\title{
Oral glutamine supplements reduce concurrent chemoradiotherapy-induced esophagitis in patients with advanced non-small cell lung cancer
}

\author{
Shih-Chieh Chang, MD ${ }^{\mathrm{a}, \mathrm{b}}$, Yi-Chun Lai, MDa , Jui-Chi Hung, MRes ${ }^{\mathrm{c}}$, Cheng-Yu Chang, MD ${ }^{\mathrm{d}}{ }^{*}$
}

\begin{abstract}
Background: Complications related to concurrent chemoradiotherapy (CCRT) such as acute radiation-induced esophagitis (ARIE) may cause significant morbidity and unplanned treatment delays in patients with advanced non-small cell lung cancer (NSCLC). We designed a prospective randomized study to assess the impact of glutamine (GLN) supplementation in preventing CCRT-induced toxicities of advanced NSCLC patients.

Methods: From September 2014 to September 2015, 60 patients diagnosed with NSCLC were included to the study. Thirty patients (50\%) received prophylactic powdered GLN orally at a dose of $10 \mathrm{~g} / 8 \mathrm{~h}$. The prescribed radiation dose to the planning target volume was 30 Gy in 2-Gy fractions. The endpoints were radiation-induced esophagitis, mucositis, body weight loss, overall survival and progression-free survival.

Results: The 60 patients with NSCLC included 42 men and 18 women with a mean age \pm standard deviation of 60.3 years \pm 18.2 (range, 44-78 years).

At a median follow-up of 26.4 months (range 10.4-32.2), all patients tolerated GLN well. A administration of GLN was associated with a decrease in the incidence of grade 2 or 3 ARIE (6.7\% vs 53.4\% for GIn+ vs Gln-; $P=.004)$. GLN supplementation appeared to significantly delay ARIE onset for 5.8 days (18.2 days vs 12.4 days; $P=.027$ ) and reduced incidence of weight loss (20\% vs $73.3 \%$; $P=.01)$.
\end{abstract}

Discussion: Our study suggests a beneficial effect of oral glutamine supplementation for the prevention from radiation-induced injury and body weight loss in advanced NSCLC patients who receiving CCRT.

Abbreviations: $\mathrm{ARIE}=$ acute radiation-induced esophagitis, $\mathrm{CCRT}=$ concurrent chemoradiotherapy, $\mathrm{ECOG}=\mathrm{Eastern}$ Cooperative Oncology Group, GLN = glutamine, NSCLC = non-small cell lung cancer.

Keywords: advanced non-small cell lung cancer, concurrent chemoradiotherapy, esophagitis, glutamine

\footnotetext{
Editor: Alfons Lawen.

We hereby confirm that full authorship and all institutional affiliations. The manuscript has not been published or submitted for publication elsewhere except as a brief abstract in the proceedings of a scientific meeting or symposium.

All authors have contributed significantly, and that all authors are in agreement with the content of the manuscript.

All procedures performed in studies involving human participants were in accordance with the ethical standards of the institutional review board and/or national research committee and with the 1964 Helsinki Declaration and its later amendments or comparable ethical standards.

Informed consent: Informed consent was obtained from all individual participants included in the study.

The authors have no funding and no conflicts of interest to disclose.

${ }^{a}$ Division of Chest Medicine, Department of Internal Medicine, ${ }^{b}$ Department of Critical Care Medicine, National Yang-Ming University Hospital, Yi-Lan, ' ${ }^{\circ}$ Division of Infectious Disease, ' Division of Chest Medicine, Department of Internal Medicine, Far Eastern Memorial Hospital, New Taipei City, Taiwan.

* Correspondence: Cheng-Yu Chang, Division of Chest Medicine, Department of Internal Medicine, Far Eastern Memorial Hospital, New Taipei City, Taiwan, No.

21, Sec. 2, Nanya S. Rd., Banciao Dist., New Taipei City.

(e-mail: koala2716@hotmail.com).

Copyright (๑) 2019 the Author(s). Published by Wolters Kluwer Health, Inc. This is an open access article distributed under the Creative Commons

Attribution License 4.0 (CCBY), which permits unrestricted use, distribution, and reproduction in any medium, provided the original work is properly cited.

Medicine (2019) 98:8(e14463)

Received: 16 August 2018 / Received in final form: 21 December 2018 /

Accepted: 17 January 2019

http://dx.doi.org/10.1097/MD.0000000000014463
}

\section{Introduction}

Concurrent chemoradiation therapy (CCRT) is one of the major therapeutic options for non-small cell lung cancer (NSCLC), especially in locally advanced disease.$^{[1-3]}$ Despite proven treatment efficacy, CCRT-related adverse events may result in significant morbidity and even treatment-related death. ${ }^{[4,5]}$ Acute radiation-induced esophagitis (ARIE) is a common complication in patients with lung or esophageal cancer receiving thoracic irradiation. CCRT remains a standard treatment option for stage IIIB NSCLC patients, but it is associated with an increase of grade $3+$ esophagitis . ${ }^{[6,7]}$ ARIE may cause significant weight loss and unplanned treatment delays.

Glutamine, one of the most abundant amino acids in many human tissues, has been demonstrated to prevent toxicity from chemotherapy and radiotherapy.$^{[8]}$ There have been few randomized trials regarding the effect of oral glutamine supplement on the incidence of ARIE in advanced NSCLC patients treated with CCRT. Therefore, we designed a prospective randomized study to assess the impact of glutamine supplementation in preventing CCRT-induced esophageal toxicities in advanced NSCLC patients.

\section{Materials and methods}

This study was conducted at Far Eastern Memorial Hospital, a 1200-bed tertiary referral medical center in northern Taiwan. Patients with advanced NSCLC(stage IIIB-IV by AJCC 
7th edition staging), who clinically met the indication for CCRT were enrolled in the study after informed consent was obtained ${ }^{[9]}$ The exclusion criteria included Eastern Cooperative Oncology Group (ECOG) performance status $\geq 2$, impaired major organ functions, symptomatic brain metastasis, and life expectancy of less than 3 months . ${ }^{[10]}$ The patients were randomized per block to either standard treatment with prophylactic oral glutamine supplement (pure left-handed glutamine 30 grams/day in 3 divided doses, every 5 grams of powder mix with $300 \mathrm{ml}$ of cold water or drink below $40^{\circ} \mathrm{C}$ ) or standard treatment. The randomization was carried out in a 1:1 ratio and using computer-generated random numbers. All others including volunteers, study staff, investigators, and data analysts were blinded to the group assignment. There was no crossover and all subjects were analyzed in an intention-to-treat manner. Enrollment was complete following recruitment of the prespecified number of subjects. The standard treatment included 2 cycles of concurrent platinum-based doublet regimen and radiotherapy. The patients who enrolled to prophylactic oral glutamine supplement group received glutamine for 1 year. The prescribed radiation dose to the planning target volume was 30 Gy in 2-Gy fractions. Body weight change was measured after completion of CCRT. Weight loss is defined more than $5 \%$ change.${ }^{[24]}$ The primary endpoint was maximal grade of ARIE and weight change. The secondary outcome was progression-free survival. The study was approved by the Institutional Review Board of Far Eastern Memorial Hospital (IRB-103029-B).

\section{Statistical analysis}

All statistical analyses were conducted using SPSS software for Windows (version22; IBM Corporation, Armonk, NY). Data are presented as frequencies for categorical variables and by mean \pm standard deviation for numerical variables. Categorical variables were compared using a chi-square test or Fisher's exact test, and continuous variables were compared using an independent unpaired $t$-test. Progression-free survival was represented by a Kaplan-Meier survival curve and calculated by a log-rank test. $P$-values of $<.05$ were considered to be statistically significant.

\section{Results}

From September 2014 to September 2015, 60 patients with newly diagnosed stage IIIB and IV NSCLC were enrolled in the study. Thirty patients $(50 \%)$ were randomized to receive oral glutamine supplements. There were 42 men and 18 women with a mean age of 60.3 years (range, 44-78). The demographic data are summarized in Table 1 . There were no significant differences between demographic characteristics between the groups, except for a male predominance in the control group. The most common histologic type of NSCLC was adenocarcinoma (45\%), followed by squamous cell carcinoma $(36.7 \%)$. Patients in the oral glutamine supplement and control groups received similar chemotherapy regimens of taxane/platinum, pemetrexed/platinum, and vinorelbine/platinum. After a median follow-up period of 26.4 months (range, 10.4-32.2), all patients tolerated the 12month oral glutamine supplements well. No grade 4-5 ARIE was observed in either group. Compared with the control group, the oral glutamine supplement group had significantly less grade 2-3 ARIE $(6.7 \%$ vs $53.3 \% ; P=.004)$. In addition, oral glutamine supplements significantly delayed the onset of grade 2-3 ARIE (18.2 vs 12.4 days: $P=.027$ ) and reduced the incidence of weight loss $(20 \%$ vs $73.3 \% ; P=.01)$. No statistically significant
Table 1

Patient characteristics.

\begin{tabular}{|c|c|c|c|}
\hline & $\begin{array}{c}\text { Glutamine } \\
(+)(\mathrm{N}=30)(\%)\end{array}$ & $\begin{array}{c}\text { Glutamine } \\
(-)(\mathrm{N}=30)(\%)\end{array}$ & $P$ value \\
\hline Age (years) & & & .602 \\
\hline$\leqq 60$ & $18(60.0)$ & $16(53.3)$ & \\
\hline$>60$ & $12(40.0)$ & $14(46.7)$ & \\
\hline Sex & & & .005 \\
\hline Male & $16(53.3)$ & $26(86.7)$ & \\
\hline Female & $14(46.7)$ & $4(13.3)$ & \\
\hline ECOG performance status & & & .592 \\
\hline 0 & $20(66.7)$ & $18(60.0)$ & \\
\hline 1 & $10(33.3)$ & $12(40.0)$ & \\
\hline Cancer type & & & .939 \\
\hline Adenocarcinoma & $14(46.7)$ & $13(43.3)$ & \\
\hline Squamous cell carcinoma & 10 (33.3) & $12(40.0)$ & \\
\hline NSCLC & $6(20.0)$ & $5(16.7)$ & \\
\hline Stage & & & .297 \\
\hline III B & $11(36.7)$ & $15(50.0)$ & \\
\hline IV & 19 (63.3) & $15(50.0)$ & \\
\hline CT regimen & & & 1.000 \\
\hline Taxane/platinum & $16(53.3)$ & $16(53.3)$ & \\
\hline Pemetrexed/platinum & $11(36.7)$ & $10(33.3)$ & \\
\hline Navelbine/platinum & $3(10.0)$ & $4(13.3)$ & \\
\hline
\end{tabular}

Glutamine (+), glutamine use; Glutamine (-), no glutamine use.

$\mathrm{ECOG}=$ Eastern Cooperative Oncology Group.

differences in progression-free survival were observed between the glutamine supplement group and the control group (8.4 months vs 7.2 months, respectively; $P=.10$ ) by the Kaplan-Meier survival curve and log-rank test (Fig. 1). The treatment outcomes are summarized in Table 2 . The median body weight change was a $1.2 \mathrm{~kg}$ gain in oral glutamine supplement group and a $4.6 \mathrm{~kg}$ loss in the control group $(P<.001)$ (Table 2$)$.

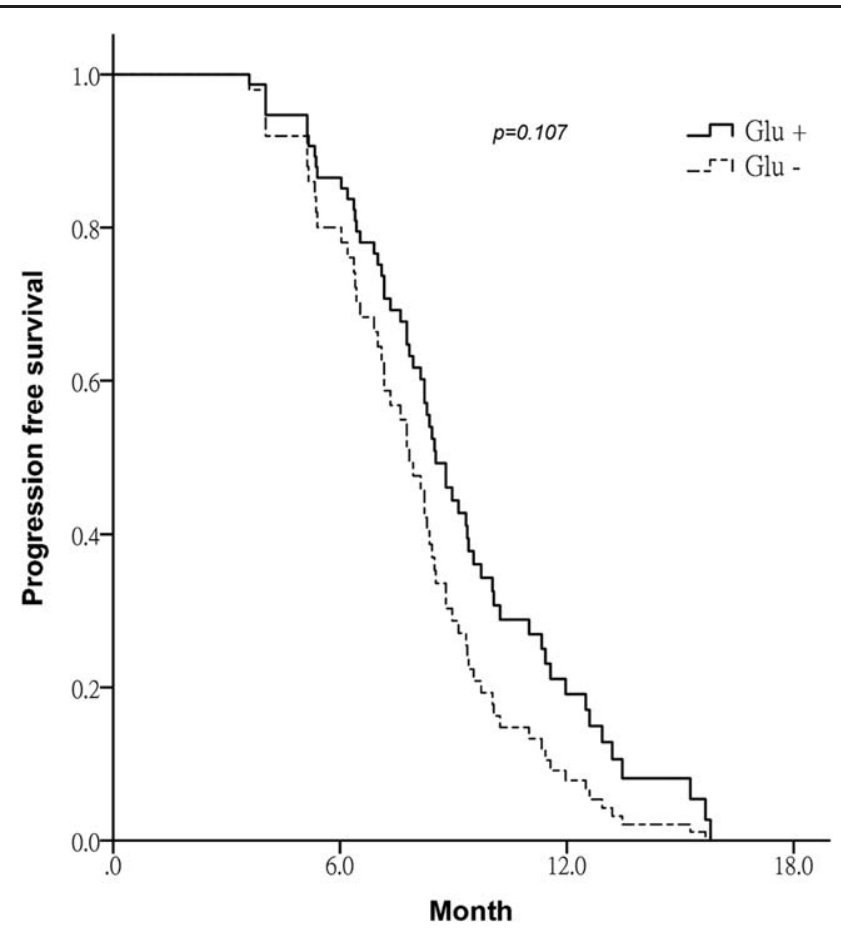

Figure 1. The Kaplan-Meier curve for advanced non-small cell lung cancer patients with or without glutamine administration. 
Table 2

Effects of glutamine use.

\begin{tabular}{|c|c|c|c|}
\hline & $\begin{array}{c}\text { Glutamine } \\
(+)(\mathrm{N}=30)(\%)\end{array}$ & $\begin{array}{c}\text { Glutamine } \\
(-)(N=30)(\%)\end{array}$ & $P$ value \\
\hline Maximum grade ARIE & & & 0.004 \\
\hline $0-1$ & 28 (93.3) & $14(46.7)$ & \\
\hline 2 & $2(6.7)$ & $10(33.3)$ & \\
\hline 3 & $0(0)$ & $6(20.0)$ & \\
\hline $4-5$ & $0(0)$ & $0(0)$ & \\
\hline Grade 2-3 ARIE onset (days) & & & 0.027 \\
\hline Median & 18.2 & 12.4 & \\
\hline Range & $11.7-25.9$ & $5.8-20.1$ & \\
\hline Treatment delay & $6(20.0)$ & $10(33.3)$ & 0.06 \\
\hline Body weight & & & 0.01 \\
\hline Gain or no change & $24(80.0)$ & $8(26.7)$ & \\
\hline Loss & $6(20.0)$ & $22(73.3)$ & \\
\hline Weight change (kg) & & & $<0.001$ \\
\hline Median & 1.2 & -4.6 & \\
\hline Range & -2.8 to 5.6 & -8.8 to 2.1 & \\
\hline
\end{tabular}

Glutamine (+), glutamine use; Glutamine (-), no glutamine use; N, number; ARIE, acute radiationinduced esophagitis; kg, kilogram.

\section{Discussion}

Our prospective, randomized, study demonstrated that oral glutamine supplements (L-glutamine $30 \mathrm{~g} /$ day in 3 divided doses) could decrease the development of severe ARIE, delay grade 2-3 ARIE onset time, and reduce the incidence of weight loss in patients with stage III-IV NSCLC who received CCRT. Glutamine is a radio-protective agent ${ }^{[8,11]}$ and several studies have reported that lung cancer patients undergoing CCRT, who are taking prophylactic glutamine supplements, have a reduced risk of ARIE.$^{[12-17]}$ The ARIE toxicity grade, weight loss, and esophageal transit times were significantly improved in the glutamine supplement group in lung cancer patients with CCRT ${ }^{[13]}$ Only 2 small patient number studies showed the reducing ARIE of advance lung cancer patients undergoing radiotherapy with glutamine supplements.$^{[13-14]}$ For unresectable stage III NSCLC, CCRT remains the standard treatment and may offer a curable opportunity with approximately $15 \% 5$-year survival rate. Compared to sequential radiochemotherapy, CCRT have an advantage in overall survival but also increase the risk of severe acute esophageal toxicity from $4 \%$ to $18 \% .{ }^{[25]}$ Oral glutamine supplements may reduce the incidence of adverse effect from esophageal injury and facilitate these patients to complete defined CCRT.

Acute radiation esophagitis often lead to dysphagia and odynophagia due to mucosal changes and effects on esophageal motility $\cdot{ }^{[18,19]}$ ARIE was not only related to body weight loss, poor nutrition, and poor quality of life but also decreased the effective treatment dose of radiotherapy, increased unplanned treatment course delay, causing decreased tumor control and survival rate.${ }^{[20]}$ Late radiation-induced esophagitis usually refer to onset more than 3 months after the completion of thoracic

Table 3

Progression-free survival based on glutamine use.

\begin{tabular}{lccc}
\hline & Glutamine (+) & Glutamine (-) & $\boldsymbol{P}$ value \\
\hline $\begin{array}{l}\text { Progression-free survival } \\
\text { Median (months) }\end{array}$ & $8.4 \pm 0.274$ & $7.2 \pm 0.602$ & .100 \\
\hline
\end{tabular}

Glutamine (+), glutamine use; Glutamine (-), no glutamine use; irradiation. Some patients experienced severe esophageal stricture and required surgical interventions.$^{[26]}$ A retrospective study demonstrated prophylactic oral glutamine supplementation reduced incidence and severity of both acute and late radiation induced esophagitis . ${ }^{[20]}$ Body weight loss is an important prognostic factor of survival in patients with lung cancer . [21] Glutamine is a neutral amino acid, which can provide host energy reserves, nutrition support in cancer cachexia, and improve radiation therapy-induced esophagitis in patients with head and neck neoplasia.$^{[13,22]}$ Glutamine is also an oxidative supply for epithelial integrity maintenance.${ }^{[8,23]}$ Several studies showed that lung cancer patients receiving glutamine supplements had significant weight gain compared with non-glutamine groups. ${ }^{[13,16]}$ Our study demonstrated the same result.

Our study has some limitations. First, the sample size was small and it was a single-center experience. However, the only two prospective studies for advance lung cancer patients undergoing radiotherapy and who received glutamine supplements to reduce ARIE had 32 cases ${ }^{[13]}$ and 46 cases.$^{[14]}$ Another retrospective study that enrolled advanced lung cancer patients undergoing CCRT, who received glutamine supplements had 102 cases, and another retrospective study ${ }^{[16]}$ for patients with stage III lung cancer undergoing radiotherapy had 41 cases. Our prospective, randomized study included 60-advanced lung cancer cases, which is the largest study focusing on ARIE in current literature. Second, although we provided glutamine to reduce ARIE, we did not record daily calorie-intake amounts in all the patients. Third, our study focused on the relationship between oral glutamine and esophagitis, the palliative CCRT of our patients including stage IIIB and stage IV were by clinical decisions. Forth, the radiation regimen is not the standard prescription doses for CCRT in advanced NSCLC. We use the 30 Gy in 2-Gy fractions as a palliative CCRT to minimize the radiation injury for Asia patients. According to the American Society for Radiation Oncology (ASTRO) guideline, ${ }^{[27]}$ a moderately hypofractionated approach with external beam radiation therapy is recommended, where "moderate" is defined as daily radiation doses of 280-300 cGy) per fraction to a total dose of 3000 or 4200 cGy. Higher radiation doses may not be tolerable for palliative-intent patients, and lower doses may not confer a quality-of-life benefit. The best practice also includes minimizing unnecessary radiation dose to the esophagus. Fifth, the radiation location, involved esophagus volume and chemotherapy regimen can also affect the incidence and degree of esophagitis. In our study, we design CCRT on primary tumor site and cannot distinguish the influence of radiation location and chemotherapy regimen due to small patient numbers.

\section{Conclusions}

In conclusion, this study demonstrated that oral glutamine supplements has a benefit in delaying onset of and decrease the severity of ARIE in advanced lung cancer patients undergoing CCRT. With oral glutamine use, the patients had improved quality of life and better treatment outcomes.

\section{Author contributions}

Data curation: Jui-Chi Hung.

Formal analysis: Yi-Chun Lai, Cheng-Yu Chang, Shih-Chieh Chang.

Methodology: Yi-Chun Lai, Cheng-Yu Chang, Shih-Chieh Chang. 
Validation: Yi-Chun Lai, Jui-Chi Hung.

Writing - review \& editing: Yi-Chun Lai, Cheng-Yu Chang, Shih-Chieh Chang.

\section{References}

[1] Albain KS, Crowley JJ, Turrisi AT3rd, et al. Concurrent cisplatin, etoposide, and chest radiotherapy in pathologic stage IIIB non-small-cell lung cancer: a Southwest Oncology Group phase II study, SWOG 9019. J Clin Oncol 2002;20:3454-60.

[2] Belani CP, Choy H, Bonomi P, et al. Combined chemoradiotherapy regimens of paclitaxel and carboplatin for locally advanced non-smallcell lung cancer: a randomized phase II locally advanced multi-modality protocol. J Clin Oncol 2005;23:5883-91.

[3] Vokes EE, Senan S, Treat JA, et al. PROCLAIM: A phase III study of pemetrexed, cisplatin, and radiation therapy followed by consolidation pemetrexed versus etoposide, cisplatin, and radiation therapy followed by consolidation cytotoxic chemotherapy of choice in locally advanced stage III non-small-cell lung cancer of other than predominantly squamous cell histology. Clin Lung Cancer 2009;10:193-8.

[4] Uyterlinde W, Belderbos J, Baas C, et al. Prediction of acute toxicity grade $>/=3$ in patients with locally advanced non-small-cell lung cancer receiving intensity modulated radiotherapy and concurrent low-dose Cisplatin. Clin Lung Cancer 2013;14:541-8.

[5] Palma DA, Senan S, Tsujino K, et al. Predicting radiation pneumonitis after chemoradiation therapy for lung cancer: an international individual patient data meta-analysis. Int J Radiat Oncol Biol Phys 2013;85:444-50.

[6] Robinson LA, Ruckdeschel JC, Wagner HJr, et al. Treatment of nonsmall cell lung cancer-stage IIIA: ACCP evidence-based clinical practice guidelines (2nd edition). Chest 2007;132:243s-65s

[7] Socinski MA, Zhang C, Herndon JE2nd, et al. Combined modality trials of the Cancer and Leukemia Group B in stage III non-small-cell lung cancer: analysis of factors influencing survival and toxicity. Ann Oncol 2004;15:1033-41.

[8] Savarese DM, Savy G, Vahdat L, et al. Corey B: prevention of chemotherapy and radiation toxicity with glutamine. Cancer Treat Rev 2003;29:501-13.

[9] Edge SB, Compton CC. The American Joint Committee on Cancer: the 7th edition of the AJCC cancer staging manual and the future of TNM. Ann Surg Oncol 2010;17:1471-4.

[10] Oken MM, Creech RH, Tormey DC, et al. Toxicity and response criteria of the Eastern Cooperative Oncology Group. Am J Clin Oncol 1982;5:649-55.

[11] Bartlett DL, Charland S, Torosian MH. Effect of glutamine on tumor and host growth. Ann Surg Oncol 1995;2:71-6.

[12] Rodriguez N, Algara M, Foro P, et al. Predictors of acute esophagitis in lung cancer patients treated with concurrent three-dimensional confor- mal radiotherapy and chemotherapy. Int J Radiat Oncol Biol Phys 2009; 73:810-7.

[13] Gul K, Muge A, Taner A, et al. Oral glutamine supplementation reduces radiotherapy- induced esophagitis in lung cancer patients. Asian Pac J Cancer Prev 2015;16:53-8.

[14] Tutanc OD, Aydogan A, Akkucuk S, et al. The efficacy of oral glutamine in prevention of acute radiotherapy-induced esophagitis in patients with lung cancer. Contemp Oncol (Poznan, Poland) 2013;17:520-4.

[15] Algara M, Rodriguez N, Vinals P, et al. Prevention of radiochemotherapy-induced esophagitis with glutamine: results of a pilot study. Int J Radiat Oncol Biol Phys 2007;69:342-9.

[16] Topkan E, Yavuz MN, Onal C, et al. Prevention of acute radiationinduced esophagitis with glutamine in non-small cell lung cancer patients treated with radiotherapy: evaluation of clinical and dosimetric parameters. Lung Cancer (Amsterdam, Netherlands) 2009; 63:393-9.

[17] Gul K, Mehmet K, Meryem A. The effects of oral glutamine on clinical and survival outcomes of non-small cell lung cancer patients treated with chemoradiotherapy. Clin Nutr (Edinburgh, Scotland) 2016;36:1022-8.

[18] Sasso G, Rambaldi P, Sasso FS, et al. Scintigraphic evaluation of oesophageal transit during radiotherapy to the mediastinum. BMC Gastroenterol 2008;8:51.

[19] Turkolmez S, Atasever T, Akmansu M. Effects of radiation therapy on oesophageal transit in patients with inner quadrant breast tumour. Nucl Med Commun 2005;26:721-6.

[20] Topkan E, Parlak C, Topuk S, et al. Influence of oral glutamine supplementation on survival outcomes of patients treated with concurrent chemoradiotherapy for locally advanced non-small cell lung cancer. BMC Cancer 2012;12:502.

[21] Stanley KE. Prognostic factors for survival in patients with inoperable lung cancer. J Natl Cancer Inst 1980;65:25-32.

[22] Huang EY, Leung SW, Wang CJ, et al. Oral glutamine to alleviate radiation-induced oral mucositis: a pilot randomized trial. Int J Radiat Oncol Biol Phys 2000;46:535-9.

[23] Kuhn KS, Muscaritoli M, Wischmeyer P, et al. Glutamine as indispensable nutrient in oncology: experimental and clinical evidence. Eur J Nutr 2010;49:197-210.

[24] Arends J, Baracos V, Bertz H, et al. ESPEN expert group recommendations for action against cancer-related malnutrition. Clin Nutr 2017; 36:1187-96.

[25] Aupérin A, Le Péchoux C, Rolland E, et al. Meta-analysis of concomitant versus sequential radiochemotherapy in locally advanced non-small-cell lung cancer. J Clin Oncol 2010;28:2181-90.

[26] Baker S, Fairchild A. Radiation-induced esophagitis in lung cancer. Lung Cancer 2016;7:119-27.

[27] Rodrigues G, Videtic GM, Sur R. Palliative thoracic radiotherapy in lung cancer: An American Society for Radiation Oncology evidence-based clinical practice guideline. Pract Radiat Oncol 2011;1:60-71. 